da wo wir Hybride mit diff-differenten Wissensressourcen spielen können, wo wir die zusammenbringen können wo wir auch- wo wir Uneindeutigkeiten bestehen lassen können, ahm wissend, dass wir- also unsere Disziplin selbst nicht eindeutig positionierbar ist oder positioniert ist.“ (T3, Z. 720-736)

Die Soziologie befindet sich im Außen, in der Gesellschaft. Normative Prinzipien sind wie im Prinzip der Inklusion auszuweisen, wobei diese überwiegend ontologisiert werden, also an die Praxis ausgelagert: Die Soziologie ist Wissenszulieferer für die Praxis, die Praxis verarbeitet die Forschungsergebnisse nach ihrer eigenen Logik. Wissenschaftliche Autorität wird hier erzeugt über die Kreativität und Innovationsfähigkeit der Disziplin und das Anwendungsprinzip, sprich über die Ergebnisse, die sie in der Praxis erzielt.

\title{
5.2 DAS IDENTITÄTSDEFIZIT DER SOZIOLOGIE
}

Wir haben bereits im Kapitel zur Geschichte der Soziologie gehört, dass die Soziologie über eine geringe kognitive Konsolidierung verfügt. Zudem stellen Autoren wie Cole (1994), Whitley (1984b); Lammers (1974) oder Stinchcombe (1994) die Ausbildung eines kognitiven Kerns der Soziologie infrage. In dieser Arbeit war die Fragmentierung der Soziologie auch Anlass, vermehrt über die Bestimmung eines geeigneten Samples nachzudenken.

Aus der Doppelstruktur der Soziologie erwächst deshalb nicht nur ein Autoritätsdefizit, sondern auch ein Identitätsdefizit. Reckwitz (2002) versteht Identität als Problem des Sinns verstanden, den Individuen und Kollektive ihrem Handeln zuschreiben. Identität bezeichnet in diesem Fall also eine „Kontingenz des Selbstverstehens“ (Reckwitz, 2002, S. 22). Identität bedeutet, sich als ,jemand“ (Reckwitz, 2002, 30) zu interpretieren. Identität kann nicht objektiv sein, sie wird immer auf die subjektive Art der Selbstinterpretation bezogen, die sich vor dem Hintergrund kollektiver Bedeutungshorizonte vollzieht. Diese werden als historisch und kulturell spezifische Wissensordnungen begriffen (Reckwitz, 2002, S. 30 f.).

Vor welchem Bedeutungshorizont also definieren sich Soziolog(inn)en? Wie schreiben sie ihrer Arbeit selbst Sinn zu? Anknüpfend an die Aussagen von Kaldewey (2013) und Calvert (2006), dass Grenzarbeit immer auch mit Identitätsarbeit verbunden ist, soll dieser Aspekt im Folgenden vertieft werden.

Wir haben im vorhergehenden Abschnitt gesehen, wie epistemische Autorität der Soziologie in Hinblick auf ihr Verhältnis zum Untersuchungsgegenstand auf unterschiedliche Weise erzeugt wird. Grenzen wurden erweitert, verengt oder 
geschützt. Wenn wir jedoch auf dieser Ebene stehenbleiben, ist der Versuch, die Sache mit der Person zu verknüpfen, noch nicht vollständig geglückt. Wir haben also im Sinn der dokumentarischen Methode sinngenetische, aber noch keine soziogenetischen Typen gebildet. Die Analyse der Interviews hat gezeigt, dass sich die Soziologie durch eine große Interpretationsoffenheit auszeichnet. Die Wege in die Soziologie waren dispers, von unterschiedlichsten Vorstellungen, Erwartungen und persönlichen Erfahrungen geprägt. Sinnkonstruktionen der eigenen Arbeit, von Wissenschaft und von der Soziologie variierten dementsprechend. Es konnte keine einheitliche soziologische Identität im Sinn einer wissenschaftlichen Identität oder einer Identität der Praxis ausgemacht werden. Sozio$\log ($ inn)en haben sich selbst mit Ausdrücken wie „Mythenjäger“ oder ,public sociologist“ beschrieben oder sich dagegen abgegrenzt, ein ,verdammter Sozialarbeiter“ zu sein. Das Identitätsdefizit der Soziologie ist, dass es im engeren Sinn keine übergreifenden Bedeutungshorizonte gibt, vor denen sich eine soziologische Identität entfalten könnte. Diese sind, entsprechend der Interpretationsoffenheit der Soziologie, multipel. Es gibt also vor dem Hintergrund der Fragmentierung und des fehlenden Kerns der Soziologie weder die Soziologie noch den Soziologen bzw. die Soziologin.

Diese Ergebnisse stehen im Gegensatz zu denen von Lam, die argumentiert, dass differierende Orientierungen von Forschenden einer ,academic identity role“, „hybrid roles“ und „dual role identities“ folgen (Lam, 2010, S. 315). Denn das würde bedeuten, dass Konsens darüber besteht, wie eine solche akademische Identität im Gegensatz zu einer hybriden Identität aussieht. Im Gegensatz dazu haben die Interviewten selbst ihr soziologisches Ich konstruiert, und zwar über Grenzziehungen der Soziologie zu ihrem Untersuchungsgegenstand und ihr Verhältnis zur Soziologie selbst. Die Beziehung zum Untersuchungsgegenstand galt jeweils als Gradmesser dafür, inwieweit man möglicherweise durch die Peers ausgeschlossen wurde. Die Frage also, wer zur Soziologie gehört und wer nicht, ist als fragil markiert worden. Diese Art von Selbstbeschreibung zeichnet sich durch ein vocabulary of identity making aus:

„Ich bin überzeugter Soziologe in gewisser Weise, auch wenn ich fast immer interdisziplinär gearbeitet hab und nich im engen Sinne Soziologe bin, ahm also Soziologen würden ablehnen, dass ich einer bin im engeren Sinne ahm aber ich sehe das (3) lockerer.“ (T3, Z. 699-702)

„Also ich hab' in der Zeit, als ich im [Name der Organisation] war, [...], hätte ich mich glaub' ich nicht als marxistischen Soziologen bezeichnet. //I: hm hm// ich mache das jetzt auch nur mit vielen Zusätzen [...]. also ich hab' jetzt nicht den Eindruck, dass ich diskri- 
miniert werde //I: hm// in der Zunft, also nee, werd' ich nicht, kann ich nicht sagen, weder bei Drittmitteln noch bei anderem, nee, werd' ich nicht. also es gibt sicher Naserümpfen und Kritik, aber ich werde nicht diskriminiert, aber ich bezieh' ne Außenseiterposition, das ist ganz klar. in der internationalen Soziologie, jetzt auf dem ISA-Kongress in Wien beispielsweise ist es ganz anders. also ich war auf dem am besten besuchten äh auf der am besten besuchten Plenary Session da mit Michael Burawoy und jetzt komm' ich zur Antwort auf die Frage und wir waren die bestbewertete Session und ich wurde dreimal von [Name der Person] gelobt [...]. also ich weiß nicht, ob ich das auf nem deutschen Soziologentag machen würde, Soziologiekongress, nicht Soziologentag, machen würde, wahrscheinlich inzwischen auch, aber das ging äh auf dem- auf diesem internationalen Kongress mit der Hälfte der Teilnehmer wunderbar. die südafrikanische Delegation kam und hat sich bedankt usw. usw., das hatte ich noch nie erlebt.“" (T9, Z. 379-392)

Diese beiden Zitate zeigen noch einmal eindrücklich, wie sich die Interviewten selbst in der (deutschen) Soziologie verorten. T3 schließt sich quasi aus der Zunft der Soziologen aus, da er argumentiert, selbst „überzeugter Soziologe“ zu sein, aber von den Soziologen abgelehnt zu werden, da er überwiegend interdisziplinär gearbeitet hat. T9 bezeichnet sich in seiner Selbstbeschreibung als ,marxistischer" wie auch „öffentlicher“ (T9, Z. 233) Soziologe. Er verweist auf den Unterschied zwischen der deutschen und der internationalen Soziologie und beschreibt, wie sich seine „Außenseiterposition“ in der deutschen Soziologie genau in das Gegenteil wandelt, wenn er sich auf der internationalen Bühne der (public) Soziologie bewegt.

Die Community ist also ein wichtiger Referenzfaktor für Selbstbeschreibungen. Personen, die sich selbst stärker im „Innen“ oder „Kern“ der Disziplin verorten, verwenden in ihrem vocabulary of identity making wesentlich weniger Legitimationsrhetorik in Hinblick auf ihre Selbstbeschreibung. Dennoch gab es ebenso Soziolog(inn)en, die sich zwar laut Selbstbeschreibung nicht im Kern der Disziplin bewegten, jedoch wesentlich weniger auf Zustimmung innerhalb der Disziplin Wert legten, wie das Zitat von T3 zeigt. Im Folgenden werden vier Typen vorgestellt, die sich eines unterschiedlichen vocabulary of identity making bedienen: die Zentristen, die Konformisten, die Individualisten und die AntiSoziologen (Tabelle 10). 
Tabelle 10: Typen soziologischer Identitätskonstruktion

\begin{tabular}{|l|l|l|l|l|}
\hline & Zentristen & Konformisten & Individualisten & $\begin{array}{l}\text { Anti- } \\
\text { Soziologen }\end{array}$ \\
\hline $\begin{array}{l}\text { Vocabulary of } \\
\text { identity ma- } \\
\text { king }\end{array}$ & $\begin{array}{l}\text { „Hüter“ der } \\
\text { Disziplin }\end{array}$ & $\begin{array}{l}\text { Rückversiche- } \\
\text { rung gegen- } \\
\text { über den Peers }\end{array}$ & $\begin{array}{l}\text { Soziologie als } \\
\text { Umsetzung in- } \\
\text { dividueller in- } \\
\text { tellektueller } \\
\text { Ziele }\end{array}$ & $\begin{array}{l}\text { Kritik am } \\
\text { Zentrum der } \\
\text { Disziplin }\end{array}$ \\
\hline $\begin{array}{l}\text { Art der Selbst- } \\
\text { beschreibun- } \\
\text { gen }\end{array}$ & $\begin{array}{l}\text { Disziplinäre } \\
\text { Selbstbe- } \\
\text { schreibungen }\end{array}$ & $\begin{array}{l}\text { Disziplinäre } \\
\text { und individu- } \\
\text { elle Selbstbe- } \\
\text { schreibungen }\end{array}$ & $\begin{array}{l}\text { Individuelle } \\
\text { Selbstbe- } \\
\text { schreibungen } \\
\text { Skepsis ge- } \\
\text { genüber } \\
\text { disziplinären } \\
\text { Selbstbe- } \\
\text { schreibungen }\end{array}$ & $\begin{array}{l}\text { Selbstbe- } \\
\text { schreibungen, } \\
\text { Abgrenzung } \\
\text { von disziplinä- } \\
\text { ren Selbstbe- } \\
\text { schreibungen }\end{array}$ \\
& $\begin{array}{l}\text { Individuelle } \\
\text { Identitätspoli- } \\
\text { tik }\end{array}$ & $\begin{array}{l}\text { Kerns, Unter- } \\
\text { stützen diszip- } \\
\text { linärer Identi- } \\
\text { tätspolitik }\end{array}$ & $\begin{array}{l}\text { len Kerns, } \\
\text { keine Teil- } \\
\text { nahme an dis- } \\
\text { ziplinärer } \\
\text { Identitätspoli- } \\
\text { tik }\end{array}$ & $\begin{array}{l}\text { Identitätspoli- } \\
\text { tik }\end{array}$ \\
\hline $\begin{array}{l}\text { Stellung in- } \\
\text { nerhalb der } \\
\text { Disziplin }\end{array}$ & $\begin{array}{l}\text { Situierung im } \\
\text { Zentrum }\end{array}$ & $\begin{array}{l}\text { Willen, am } \\
\text { Zentrum teil- } \\
\text { zunehmen }\end{array}$ & $\begin{array}{l}\text { Distanzierung } \\
\text { vom Zentrum }\end{array}$ & $\begin{array}{l}\text { Situierung an } \\
\text { den Rändern }\end{array}$ \\
\hline $\begin{array}{l}\text { Verhältnis zur } \\
\text { Disziplin }\end{array}$ & $\begin{array}{l}\text { Konsolidie- } \\
\text { rung eines } \\
\text { disziplinären }\end{array}$ & $\begin{array}{l}\text { Konsolidie- } \\
\text { rung eines } \\
\text { disziplinären }\end{array}$ & $\begin{array}{l}\text { Kein Beitrag } \\
\text { zur Festigung } \\
\text { derns, starke }\end{array}$ & $\begin{array}{l}\text { Skepsis gegen- } \\
\text { über disziplinä- } \\
\text { rem Kern, }\end{array}$ \\
\hline
\end{tabular}

\subsubsection{Zentristen}

Disziplinäre Referenzfolien haben für das vocabulary of identity making von Zentristen einen besonders hohen Stellenwert. Zentristen sehen sich selbst als Hüter der Disziplin und wollen ein Zentrum der Disziplin definieren. Ihre Selbstbeschreibung wird von einer Rhetorik geprägt, die sich nicht über individuelle, sondern über disziplinäre Selbstbeschreibungen auszeichnet. Die Reichweite ihrer Argumentation steht deshalb für die ganze Disziplin und nicht als individuelle Meinung. Sie betreiben eine starke Identitätspolitik, und die Grenzen, wer Zugang zur Disziplin haben sollte und wer nicht, werden deshalb auch streng kontrolliert und klar markiert. Zentristen befinden sich dementsprechend 
an gefestigten Positionen in der Disziplin. Ihnen geht es insbesondere darum, eine gemeinsame Sprache für die Soziologie zu finden und scharfe Grenzen sowohl zu anderen Formen der Deutung des Sozialen zu ziehen als auch zu anderen Disziplinen.

„Ich glaube eher, wir können- wir können unsere Karte eher dadurch spielen, dass wir unsere Disziplinarität betonen, ich finde es eher besser. Also wir sollten eher Differenzeffekte erzeugen, zu sagen okay, ihr sagt das so //I: hm// von unserer soziologischen Auffassung her sieht das aber so aus, auch wenn ich mit Künstlern zu tun habe, ist immer- [...] Künstler, ähm auch äh äh Leute, Fotografen usw., also war von Anfang an in der Forschung mit drin, hab' mit denen viel Streit gehabt //I: hm// hab ich gesagt, das könnt ihr doch nicht sagen, das stimmt doch gar nicht, was ihr da sagt und dieser Streit war produktiv, weil ich dann irgendwann gemerkt hab', die haben aber noch andere Dimensionen, andere Perspektiven, die ich nicht habe, //I: hm// die hab' ich aber erst aus dem Streit herausgekriegt //I: ja ja, hm hm// und deshalb bin ich kein Freund dieser schnellen In- äh Interdisziplinarität, bin eher einer, der sagt, lasst uns das Gespräch über Differenzen führen und nicht, wo wir Grenzen überschreiten können.“ (T8, Z. 771-785).

\subsubsection{Konformisten}

Konformisten zeichnen sich im Gegensatz zu den Zentristen - unabhängig von ihrer Position - durch eine große Unsicherheit über ihre Stellung gegenüber den Peers aus. Sie wollen am Zentrum der Disziplin partizipieren und legitimieren sich rhetorisch über disziplinäre Referenzfolien. Der Bezug zu Theorien, bedeutenden Soziologen oder auch direkten Peers spielt in den Selbstbeschreibungen eine große Rolle. Haben Konformisten einen gesellschaftsverändernden Anspruch, so wurde meist auf individueller Ebene für diesen argumentiert und nicht auf die gesamte Disziplin bezogen. Rhetorisch ist dieser Anspruch mit hohem Rechtfertigungspotenzial verbunden und führte dementsprechend zu Ängsten der Exklusion von anderen Fachkolleg(inn)en. Die Legitimation des eigenen Tuns gilt also weniger etwaigen Kritikern außerhalb der Wissenschaft als potenziellen Fachkolleg(inn)en. Grenzen werden deshalb insbesondere innerhalb des eigenen Diskurses formuliert und reformuliert. Das eigene Tun wird insbesondere auf mögliche Kritik der Fachkolleg(inn)en hin gerechtfertigt. Letztlich findet ein vocabulary of identity making seinen Ausdruck in der Rückversicherung über disziplinäre Standards. 


\subsubsection{Individualisten}

Die Individualisten hingegen besitzen meist eine gefestigte Stellung im Feld und sind dementsprechend unabhängig von potenziellen Kritikern. Die Anerkennung von Peers ist für sie zweitrangig. Ein vocabulary of identity making dient vielmehr der Konstruktion des soziologischen Selbst, durch das sie die Vorstellung der eigenen intellektuellen Ziele umsetzen können. Die Interpretationsoffenheit der Soziologie dient deshalb als Referenzfolie für die Umsetzung individueller Vorstellungen, die überwiegend intellektueller Natur sind.

„Hmm, ja, also ich hab ähm (3), wie soll ich sagen, das- äh äh also ich hab mich schon, ich- ich hab mich gesehen, also das Selbstbild sozusagen und auch das Wunschbild von mir, also das, was ich aus mir machen wollte und wie ich mich selber gesehen habe, äh als ich Anfang 20 war- war und ähm ähm und in der späteren Zeit, weil ich- ich wollte in irgendeiner Weise eine politisch intellektuelle Existenz führen. ähm das- das- es- es gab dann auch mal sozusagen so eine- so eine äh äh Pickel in meiner Seele, die von sich dachte, das müsste vielleicht dann auch die Schriftstellerei sein, dann war's der Journalismus, ähm dann war es eben sozusagen auch natürlich alles, was- ähm also ähm ich hab dann natürlich frühzeitig irgendwie die Autoren der Frankfurter Schule gelesen usw. usf. also das heißt, ja, also eine- eine- eine politisch-intellektuelle Existenz zu führen, wobei sich das dann, also ich war ein- ich war ein politischer Aktivist seit ich 14 war und äh also etwas, was man im Französischen ein- einen- einen Militanten nennt, ja. äh dieses Militante hat sich dann so- so mit Anfang 30 äh eher verflüchtigt, sag ich mal, ähm und ähm und ja, aber es war immer schon sozusagen, da war das Bild so, also das an den Kämpfen beteiligten Intellektuellen sag ich mal und es war natürlich klar, also das ähm zumal dann, also wir sprechen eben über- über dann die 80er-Jahre in Westberlin, diese Enklave, ähm wir waren alle gut finanziert, ja. [...] wir- wir haben alle unsere Möglichkeiten gehabt und das heißt, die Wissenschaft war für mich eine- eine erwünschte und gute Gelegenheit, äh ähm das, was ich mir unter einer intellektuellen Existenz vorstellte, verwirklichen zu können. //I: $\mathrm{mhm} \mathrm{mhm} / /$ und so bin ich die Sache zunächst auch einmal angegangen, das heißt ähm ähm (2) so, dass ich ähm (2). meine ersten soziologischen Texte hab ich- hab ich eher also für den Merkur geschrieben, äh für die taz geschrieben, ähm taz spielte damals ne große Rolle, die war eine- also die war schon zu meiner Studentenzeit äh ist sie gegründet worden, ich war teilweise an- an zwei der Redaktionen mit beteiligt in der Anfangszeit und äh und dann von daher kam da, also hab ich relativ häufig auch was für die taz gemacht, gar nicht immer unter meinem Namen, ja, ähm ähm und ähm und äh so und später- später hab ich also ähm (2), also ich hab viel- ich hab gar nicht in referierten Peer Review Journals usw., da hab ich erst mal gar nicht, sondern ich habe in Kulturzeitschriften, in allen möglichen Sachen hab ich irgendwie was gemacht, ähm ähm und hab dann aber (2) ähm (2) hab 
dann schon äh schon gemerkt, also es gab dann auch- es gab sehr positive Reaktionen damals auch aus dem etablierten soziologischen Bereich, ja, äh und diese positiven Reaktionen haben mich dann sozusagen eher auch in den etablierten Bereich der Soziologie hineingezogen.“(T11, Z. 170-231)

Die Selbstbeschreibung orientiert sich deshalb überwiegend an der individuellen Ebene. Der Ausschluss wird nicht gefürchtet, vielmehr wird eine konsensuelle Definition dessen, was die Soziologie ist bzw. sein kann, abgelehnt und die Peers im Feld werden tendenziell kritisch gesehen. Es wird deshalb auch kein fundamentaler Beitrag zu einer disziplinären Identitätspolitik geleistet.

\subsubsection{Anti-Soziologen}

Anti-Soziologen situieren sich - unabhängig von ihrer aktuellen Position - außerhalb der Soziologie bzw. an deren Rändern. Obwohl sie sich als Sozio$\log (\mathrm{inn})$ en begreifen, grenzen sie sich ab von ihren Peers und dem, was sie als Mainstream erfahren. So hat sich der wohl bekannteste Anti-Soziologe Helmut Schelsky von einer „Priesterherrschaft der Intellektuellen“ (1975) distanziert, die er damals als die dominante Art von Soziologie erfahren hat (siehe Kapitel 2.4). Das vocabulary of identity making übt Kritik am Zentrum der Disziplin. Letztlich sind es der distanzierte Blick auf die Disziplin und die explizite Situierung außerhalb eines Zentrums wie auch die Kritik am wahrgenommenen Zentrum, mit denen die Anti-Soziologen ihre Identitätskonstruktion vornehmen. AntiSoziologen grenzen sich dezidiert von etablierten disziplinären Selbstbeschreibungen $a b$. Sie versuchen vielmehr, eine andere Identitätspolitik als die bestehende zu betreiben.

\subsection{DAS INNOVATIONSDILEMMA DER SOZIOLOGIE}

Was sagen das Autoritätsdefizit und das Identitätsdefizit über die Soziologie selbst und ihr Verhältnis zu ihrem Untersuchungsgegenstand aus? Zunächst zeigt sich, dass die Autorität und die Identität der Disziplin als prekär anzusehen sind und von den Mitgliedern der Disziplin in unterschiedlicher Weise konstruiert werden. Soziologie wird zu einem ,essentially contested concept“ (Gallie, 1956), das aufgrund der volatilen Grenzen ,in the making“ ist. Ursprünglich wurden diese concepts von Gallie auf die Kunst oder die Demokratie angewandt. Sie sind als semantische Konstruktionen anzusehen, in denen es einen Interpreta- 